\title{
Tangence
}

\section{La nouvelle : un art urbain ?}

\section{Lise Gauvin}

Numéro 48, octobre 1995

Montréal et Vancouver : parcours urbains dans la littérature et le cinéma

URI : https://id.erudit.org/iderudit/025869ar

DOI : https://doi.org/10.7202/025869ar

Aller au sommaire du numéro

Éditeur(s)

Tangence

ISSN

0226-9554 (imprimé)

1710-0305 (numérique)

Découvrir la revue

Citer cet article

Gauvin, L. (1995). La nouvelle : un art urbain ? Tangence, (48), 147-158.

https://doi.org/10.7202/025869ar

Ce document est protégé par la loi sur le droit d'auteur. L'utilisation des services d'Érudit (y compris la reproduction) est assujettie à sa politique d'utilisation que vous pouvez consulter en ligne.

https://apropos.erudit.org/fr/usagers/politique-dutilisation/
Cet article est diffusé et préservé par Érudit.

Érudit est un consortium interuniversitaire sans but lucratif composé de l’Université de Montréal, l'Université Laval et l'Université du Québec à Montréal. Il a pour mission la promotion et la valorisation de la recherche. https://www.erudit.org/fr/ 


\section{La nouvelle: un art urbain? Lise Gauvin}

Il semble aller de soi de lier la modernité littéraire, telle que nous la concevons aujourd'hui, à la ville, plus particulièrement à la ville industrielle du dix-neuvième siècle. La cité moderne en effet modifie aussi bien l'horizon des genres que les figures de l'écrivain et les représentations de la littérature. Depuis Baudelaire, l'image de l'écrivain est celle d'un homme se frayant un chemin au milieu de la foule et tâchant, partagé entre l'euphorie et la dysphorie de la ville, de transformer en signes lisibles les ondes de choc qu'il en reçoit. Ainsi le phénomène de la masse serait à l'origine de l'art moderne. C'est, selon Benjamin, "une matrice d'où sort tout un ensemble d'attitudes nouvelles à l'égard de l'œuvre d'art" ${ }^{1}$. Le même critique découvre chez Baudelaire, "presque partout, la présence mystérieuse de la foule" ${ }^{2}$, et ce même si le poète n'en a pas fait un objet de description directe. La figure de l'écrivain recoupe alors celle du flâneur: "Le flâneur ne voit pas les choses telles qu'elles sont, mais telles qu'elles lui conviennent. Il ne veut pas admettre qu'il s'agisse d'une masse uniforme, se mouvant mécaniquement, qui le croise dans les rues: il la transfigure en une foule dotée d'une âme. La foule est le voile à travers lequel la ville qui lui est familière, en tant que fantasmagorie, fait signe au flâneur", écrit Walter Benjamin. Dans cette fantasmagorie, elle est tantôt le paysage, tantôt la chambre ${ }^{3}$.

\section{Ville, texte, modernité}

Le flâneur est celui qui regarde, celui à qui la ville fait signe, celui qui tente de lire le texte de la ville. Parmi les lieux qui s'offraient de façon privilégiée à la lecture du flâneur, au xIX

1 Walter Benjamin, "L'œuvve d'art à l'ère de la reproductibilité technique ", dans Poésie et Révolution, Denoël, 1971, p. 205, cité par Rolf Tiedemann, Études sur la philosopbie de Walter Benjamin, Arles, Actes Sud, 1987, p.104.

2 Walter Benjamin, Charles Baudelaire, Paris, Payot, 1982, p.168.

3 Walter Benjamin, Passagen-Werk, cité par Rolf-Peter Janz, "Expérience mythique et expérience historique au xix ${ }^{e}$ siècle ", dans Walter Benjamin et Paris, Éditions du Cerf, 198, p. 458. 
siècle, étaient les "passages", sortes d'îlots dans le Paris de cette époque et microcosme de la société de production-consommation. Un lieu, des objets, un toit de fer et de verre laissant apercevoir les étoiles, la foule des passants, n'est-ce pas là l'architecture même d'une forme littéraire qu'on appelle roman? $\mathrm{Au} \mathrm{XIX}^{\mathrm{e}}$ siècle, affirme Claude Duchet, "le texte se fait ville: se ramifie en réseaux de sens, augmente sa population en personnages, tend à brouiller la linéarité du récit. Le roman naît avec la ville qui réorganise à son profit le champ littéraire". Et encore: "Comme totalité signifiante, la ville est en symbiose avec le discours romanesque. La ville y décuple les possibles narratifs et légitime une topique de la rencontre, du secret, de l'interdit. Les trajets y sont toujours aléatoires. [...] Passant de la ville, le romancier est passeur de mots, essentiel artisan de ce grand passage" qu'est la littérature ${ }^{4}$. Ainsi le roman se fait porteur d'un regard urbain sur les choses.

La vie cadencée de la ville s'accompagne d'un fractionnement du temps. Ce fractionnement en une suite d'instants innombrables et uniformes est un autre indice de la modernité telle que l'a perçue Proust dans l'ouvre de Baudelaire: "Le monde de Baudelaire est un étrange sectionnement du temps", dit Proust (cité par Benjamin) ${ }^{5}$. Chaque instant peut dès lors être le moment de la plus grande chance, de la plus grande émotion. Telle est la sensation du moi dans le sonnet "À une passante" de Baudelaire. Ce sonnet présente l'image du choc telle qu'elle caractérise la vie dans la foule: "Bien loin que, pour cette apparition (la passante) qui fascine l'habitant de la grande ville, la foule ne soit qu'un antagoniste, un élément adverse, c'est elle, au contraire, qui la présente au poète. " 6

Burton Pike a bien distingué cette modification dans la représentation de la ville vers la fin du $\mathrm{xIX}^{\mathrm{e}}$ siècle, qui passe du stéréotype d'une ville statique, figée, à celui de la ville en mouvement:

L'anonymat, la discontinuité de la durée, la recherche effrénée d'une fortune rapide, l'isolement des individus et des groupes, la fragmentation et l'incohérence de l'expérience constituent le paysage mental du texte urbain depuis Baudelaire. À la suite de ce dernier, les écrivains du xx ${ }^{\mathrm{e}}$ siècle (Kafka, Thomas Mann, Musil) confrontent l'individu à la masse urbaine et rendent

4 Claude Duchet, colloque "Montréal, le grand passage", octobre 1992.

5 Charles Baudelaire, op. cit., p. 188.

6 Ibid., p. 169. 
compte d'une déshumanisation sociale, ainsi que d'un affaiblissement de la conscience collective. À l'heure contemporaine, les usagers perçoivent de moins en moins la ville en termes d'espace: "In our modern Western cities, we live in space but think in time." Soumis au temps, à la mode, au sentiment de l'éphémère, l'écrivain-citadin produit des textes où alternent et s'entre-regardent l'euphorie et la dysphorie, l'ordre et le désordre: la ville apparait à la fois comme "mighty heart and paved solitude", "nowhere city and utopia". ${ }^{7}$

Depuis Baudelaire, la modernité créatrice s'attache à saisir la vie dans ce qu'elle a de fugace et de passager, de façon à extraire de l'anonymat des foules la magie du particulier. Face à la "tyrannie de la circonstance", l'écrivain s'intéresse aux instants privilégiés, aux moments fragiles qu'il tente de rendre avec acuité. Ce n'est pas par hasard que la photographie s'est développée à peu près dans le même temps que la cité moderne.

Ce qui vient d'être dit des rapports entre ville et roman ne s'applique-t-il pas davantage et avec plus de justesse encore à la nouvelle? N'y a-t-il pas entre les poétiques de la ville et celle de la nouvelle des coïncidences troublantes? Comme le roman, la nouvelle telle que nous la connaissons aujourd'hui naît au $x^{e}{ }^{e}$ siècle. Certes, la nouvelle existait bien avant. Mais on désigne souvent sous ce nom des récits-cadres plus proches de la tradition du conte écrit ou de la nouvelle contée, comme les cent nouvelles nouvelles ou l'Heptaméron, que de la nouvelle proprement dite ${ }^{8}$. Le $\mathrm{xIX}^{\mathrm{e}}$ siècle est l'âge d'or de la nouvelle. Tous les grands romanciers ou poètes, à un moment donné, composent des nouvelles: Dumas, Balzac, Vigny, Hugo, Musset, Gautier, Stendhal, Sand. Des nouvellistes existent, qui se spécialisent dans ce genre: Mérimée, Nodier, Méry, Maupassant enfin, dont la palette extrêmement riche explore toute la gamme des récits courts. Des revues les publient (La revue de Paris, La revue des deux Mondes, etc.), sans parler des journaux.

7 Ce résumé est de Pierre Popovic dans "De la ville à sa littérature", document du groupe de recherche Montréal imaginaire, Université de Montréal, 1988, p. 14.

8 Sur ces distinctions, cf. René Godenne, La nouvelle française, PUF, 1974, 176 p. Également, J. Demers et Lise Gauvin, "Frontière du conte écrit: quelques loups-garous québécois", Littérature/ Les contes, oral/écrit, théorie, pratique, $\mathrm{n}^{\circ}$ 45, février 1982, Paris, Larousse, p. 5-23. Ainsi que de J. Demers, "Nouvelle et conte; des frontières à établir", Colloque "La nouvelle : écriture(s) et lecture(s), Collège Glendon, Toronto, 14 novembre 1992. 
La nouvelle a, selon moi, partie liée avec la modernité urbaine. À l'origine de la nouvelle, il y a l'idée de quelque chose qui passe, qui se passe. La ville prédispose à l'imprévu, à la rencontre. L'imprévu à la campagne prend souvent des proportions illimitées. On l'appelle naufrage, viol, présence du diable. L'imprévu est amplifié par le chour des résonances collectives qu'il réveille et met en action. L'imprévu tourne facilement à l'accident ou à la catastrophe. Sans trop qu'on s'en aperçoive, il s'enfle jusqu'à des proportions exemplaires qui lui donnent des allures de destin. Ou plus simplement de conte. Qu'on se souvienne des Contes pour un bomme seul d'Yves Thériault où chaque fait est amplifié par une sorte d'éclairage fatal et définitif. Dans l'univers du conte, l'imprévu n'existe pas vraiment puisque chaque élément, chaque fait est programmé en fonction d'un événement global qui se clôt à la fin du récit. Dans la nouvelle, l'imprévu reste dans les limites de l'incident, du cas, du particulier. $\mathrm{Au}$ sens étymologique d'ailleurs, "noveler" c'est "changer, renouveler ou bien se renouveler" 9 . L'imprévu de la nouvelle est une surprise, qui peut rester telle jusqu'à la fin et demeurer à l'état de question.

La disposition d'esprit particulière du nouvelliste est celle de l'attente, de la disponibilité. Tel le flâneur, le nouvelliste arpente les lieux propices à l'observation des individus. Tel le photographe, il happe les visions fugitives sur lesquelles il greffe les intrigues les plus rocambolesques. L'auteur de nouvelles perçoit et sent avant de développer son sujet. André Major a bien décrit cette gestation:

La matière première d'une nouvelle provient de choses vues ou d'anecdotes, écrit-il. [...] Ma nouvelle "La Folle d'Elvis" est née d'un petit fait vécu; en sortant de mon travail, un jour d'automne venteux, j'avais aperçu sur un banc de parc une jeune fille au regard perdu qui croquait une pomme. Un détail comme celui-là a déclenché quelque chose d'obscur en moi, qui m'a poussé à travailler.. Dans le roman, il y a nécessairement une intrigue ou une trame quelconque. Dans la nouvelle - et Tchekhov illustre bien ce phénomène - il n'y a parfois pas d'intrigue à proprement parler, mais plutôt une

9 Roger Dubuis, "La genèse de la nouvelle en France au Moyen-Âge ", Cabiers de l'association internationale des Êtudes francaises, $\mathrm{n}^{\circ} 18$, mars 1966, p.1011.

10 "Entretien", XYZ, vol. I, nº 2, étê 1985, p. 4-5. 
atmosphère, un dialogue ou une image, source d'inattendu. Le roman, lui, obéit à une direction plus claire, mieux définie. Pour ma part, jignore où je vais quand j'écris une nouvelle. Je sais seulement qu'un personnage ou une situation m'intéresse, sans que je puisse les exploiter longuement. Je ne me dis pas: " Tiens, si je développais ça, ça ferait un roman. "Non. les choses s'imposent plutôt à la vitesse de l'éclair et, généralement, j'en fais une nouvelle sans savoir au juste pourquoi. ${ }^{10}$

Qu'est-ce que le sujet d'une nouvelle? N'est-ce pas essentiellement une question de rapport, d'équilibre entre un lieu, des personnages, en quantité limitée, et une situation? Situation qui, à la différence du conte, n'a pas à être développée en événement. Selon que l'on accentue l'un ou l'autre de ces éléments de base, l'éventail des possibilités est sans fin. La nouvelle est un genre caméléon, presque autant que la lettre, et se modèle aussi bien sur le fait divers, sur l'instantané-tableau, sur le portrait, comme dans Ces enfants de ma vie de Gabrielle Roy que sur le journal ou le scénario, comme dans Sans cour et sans reproche de Monique Proulx. Ce qui se passe dans la nouvelle est souvent fort ténu. Née de la surprise et de l'imprévu, la nouvelle est toujours un "Incident de frontière". Ceci est le titre du recueil d'André Berthiaume qui, en préface, réfléchit sur son art: "Travail de tous les sens. Constellation d'images furtives à fixer. Ce serait trop dommage de les laisser s'envoler. Gouttière qui déborde, branche de merisier cassée par la tempête, champs fauves et lisières dorées. Instantanés, notations qui parlent, qui disent quelque chose de la vie oubliée, négligée. À côté des grands projets, des dogmes, des voies rapides, des enseignes au néon, des gratteciel. Petit bois nocturne pour que dure la braise." 11 Avant d'être un sujet, une fable, une intrigue, la nouvelle est une forme en mouvement.

Sans doute la nouvelle n'est pas nécessairement urbaine et le danger est ici de généraliser à l'excès. Mais le flot d'images que lui apporte la ville lui fournit une matière de premier choix. Non pas la ville bétonnée et fixée des immeubles et gratte-ciel mais celle des consciences constamment provoquées et piégées par un quotidien fertile en histoires. À peine suffit-il d'un léger déplacement du point de vue pour que le récit ait lieu, ait eu lieu. C'est ce qui arrive dans une nouvelle d'André Berthiaume qui joue sur 
152

le double sens du mot "nouvelle". Il s'agit d'un texte portant sur la lecture du journal et intitulé à juste titre "Le quotidien". Un homme, tout en sirotant son café, s'absorbe dans l'inventaire des faits divers et nouvelles du jour. Puis il s'aperçoit à sa stupéfaction qu'il s'est trompé puisqu'il s'agissait du journal de la veille: "Il ne put réprimer un long soupir de découragement. Tout ce qu'il venait de lire prit aussitôt un drôle de goût. Il se croyait un homme renseigné, il venait de perdre son temps à lire des vieilleries. La journée commençait mal. " ${ }^{12}$ Récit minimaliste s'il en est un. Alors que le conte est une métaphore avouée du quotidien, qui accumule les procédés de vêridiction pour mieux accentuer sa propre fausseté, la nouvelle y renvoie plus directement.

Frontières donc entre le réel et l'irréel, le vrai et le faux, l'intérieur et l'extérieur. Dans le texte qui vient d'être cité, le journal se fait le porte-parole de la rumeur du monde. Ainsi affichet-on du même coup les conditions de lisibilité de la nouvelle qui, au départ du moins, se nourrit du réel et le vampirise jusqu'à le faire basculer dans la pure écriture. Car la nouvelle est un perpétuel va-et-vient entre la singularité du texte et la banalité du propos, ou entre la singularité du cas et la transparence du texte. Ce qui est en jeu est rien de moins qu' "une image de la vie". Dans la nouvelle d'André Major ainsi intitulée, le personnage est un artiste qui "dessinait pour ne pas perdre la main, comme il disait, mais aussi dans l'espoir de voir surgir cette image de la vie qui l'apaiserait enfin. "13 Phénomène de littérature, la nouvelle fuit l'éloquence et lui tord le cou. L'éloquence, c'est-à-dire une certaine forme de prose poétique boursouflée et vide. Écrire des nouvelles, c'est pratiquer une forme d'écriture sans filet, sans l'appui de l'histoire à raconter à tout prix.

Si l'histoire est jusqu'à un certain point prétexte de la nouvelle, les personnages, à leur tour, sont des "passants". ${ }^{14}$ Des gens ordinaires, tout le contraire des héros. Vous, moi, n'importe qui. "Il avait l'impression d'être n'importe qui, ni meilleur ni pire qu'un autre", écrit André Major à propos d'un de ses protagonistes. ${ }^{15} \mathrm{Le}$ lecteur n'a pas besoin d'en savoir beaucoup sur les personnages.

12 Ibid., p. 114.

13 André Major, La folle d'Elvis, Montréal, Québec/Amérique, 1981, p. 47.

14 C'est la définition qu'en donne Claire Martin dans $X Y Z$, vol. I, $\mathrm{n}^{\circ} 1$, printemps 85, p. 5 .

15 André Major, La folle d'Elvis, op. cit., p. 38. 
Il ne connaît ni leurs antécédents ni leur généalogie. Le personnage de nouvelle est cadré en fonction de la situation qu'il vit, en interaction avec des lieux et des objets. Le nouvelliste est ainsi exempté du souci biographique mais également de la profondeur psychologique. Ses personnages, qu'ils soient actifs ou méditatifs, apparaissent soudainement dans son champ de vision et en disparaissent aussi brusquement. Au lecteur d'imaginer la suite.

A-t-on besoin d'ajouter que la nouvelle est l'art par excellence du fragment? De portions d'univers et de fragments de récits qui sont autant de coupes prélevées sur l'ordonnance du monde et que le narrateur, discret et peu évaluatif, propose à l'intelligence du lecteur. "Comme totalité signifiante, la ville est en symbiose avec le discours romanesque", affirme Claude Duchet. Mais n'est-on pas justifié de dire que la ville nord-américaine, Montréal par exemple, est une ville non totalisable, dont l'appréhension n'est possible que par morceaux, bribes et micro-récits? Toute l'attention du narrateur de nouvelles consiste à décrire, à nommer sans alourdir, à faire voir sans expliquer. Alors que le narrateur du conte est une sorte de deus ex machina qui affirme constamment son pouvoir sur le récit, celui de la nouvelle disparaît pour mieux faire entendre la rumeur publique.

\section{Le promeneur de Montréal}

Quelle image de la ville offre des recueils de nouvelles qui ont Montréal pour cadre privilégié?

Les nouvelles du recueil intitulé Les Montréalais d'Andrée Maillet utilisent abondamment les scènes de rue. Le texte intitulé * Pleure, pleure" débute ainsi : "À midi, la circulation s'intensifie: les véhicules se suivent de très près, les camions rouges, les taxis jaune et noir des vétérans, les autos de toutes marques, les autobus qui s'arrêtent en glissant et grinçant; on a du mal à traverser la rue Sherbrooke entre l'avenue Atwater et l'avenue Victoria; plus à l'est, n'en parlons pas, elle est bloquée." ${ }^{16}$ La position par excellence du nouvelliste est celle du "promeneur de Montréal", titre de l'une des nouvelles dans laquelle le narrateur-personnage constate: "Il n'est rien d'aussi charmant que la rue. Les gens s'y

16 Andrée Maillet, Les Montréalais, 1963, Montréal, l'Hexagone, "Typo", 1987, p. 15. 
154

croient en sécurité, incognito. Ils portent généralement leur figure propre; ils parlent franc de ce dont ils n'oseraient parler chez eux. Même quand ils se taisent, je les dérobe, je les devine ou je les invente. Cependant, il est le plus souvent inutile de les imaginer, tant ils sont transparents dès qu'ils se croient seuls, et si je n'ai pas fait cette découverte-là le premier, je l'ai, en revanche, faite sans l'aide de personne." ${ }^{17}$ La foule qui fréquente les rues est une foule "imprécise", aux mille métiers possibles: "Samedi soir, rue Saint-Laurent - les spasmes du néon, les odeurs de saucisses, de patates frites et de gefiltefish - l'homme n'est jamais isolé. J'aime à me l'imaginer capitaine ayant livré son fret, matelot, maquereau, commerçant: il en est mille espèces, ou hommesandwich, ou "collet blanc". L'homme, enfin, est prince, maître, rue Saint-Laurent, le samedi soir. Il est homme à tout faire. Il est mâle ou femelle, ou bien a oublié tout ce qu'il fut, et vieux, mendie sans foi, pour voir s'il y a encore des fous parmi les sages qui se fourvoient. " ${ }^{18}$ Ainsi défilent les personnages de mendiants, de bourgeois, de jeunes hommes, etc. L'auteur les suit jusque dans leurs chambres à coucher: en fin de recueil, des récits donnent un aperçu ironique des "mours amoureuses de cinq Montréalais"

Dans La folle d'Elvis d'André Major, la perspective est moins ethnologique ou moins figurative. Les nouvelles sont davantage appliquées à dire l'angoisse de personnages ordinaires, sur fond d'anonymat urbain. La nouvelle éponyme relate une rencontre au cours de laquelle le protagoniste s'aperçoit qu'il est interchangeable. Sous la banalité apparente du propos se profilent des images de vie, de mort et de destin. Le récit y fait figure de "dernière chance" avant la disparition finale. Ici la ville "neutralise", généralise l'expérience commune. Quant aux lieux comme tels, ce sont les parcs, les cafés, les gares, tous lieux qui reviennent aussi dans les recueils collectifs Montréal des écrivains et Nouvelles de Montréal. ${ }^{19}$

Ces textes de commande offrent de Montréal une image multiple, difficile à saisir, du Montréal babélique du récit d'Antonio D'Alfonso au Montréal "pas assez" de Gaëtan Brulotte, les uns comme les autres éprouvent une certaine difficulté à phantasmer

17 Ibid., p. 68.

18 Ibid., p. 70-71.

19 Montréal des écrivains, l'Hexagone, "Typo", 1988, 222 p. Nouvelles de Montréal, sous la direction de Micheline La France, l'Hexagone, "Typo", 1992, 250 p. 
la ville. "Très vite, vous êtes délivré du fardeau de votre identité, si gratifiant soit-il, constate le personnage de Lise Harou. Qui êtes-vous sinon un passant parmi tant d'autres, intéressé, curieux, attentif? Un figurant peut-être. ${ }^{20}$ Pour Nicole Brossard, la ville est un espace de désir:

Car une ville est un lieu qui allège et abrite l'intensité. Une ville toujours quelque part ranime en nous le désir en autant de saisons, les pluies, le singulier, le foisonnement des pensées, la mort recommencée et mille distances à parcourir qui nous font souvenir de la sueur et du froid. [...] Ma ville est multiple. Je ne sais pas mettre de clôture entre les quartiers, les parkings étalés du centre-ville. La tentation des terrasses est permanente. Je ne sais pas choisir entre les parcs, les cinémas, les façades anciennes, la saleté et les restos chic. Je sais seulement marcher entre les saisons, la tête haute, le regard neuf comme si Montréal était une aventure toujours à recommencer. ${ }^{21}$

"Une aventure toujours à recommencer". C'est comme si la ville ne préexistait pas à l'écriture. Comme si l'écriture seule lui donnait son poids de réalité. Cette impression d'une ville à choisir parmi plusieurs modes d'existence possibles se confirme encore dans le texte de Suzanne Jacob:

Supposons que tu débouches de la montagne et que tu prennes la rue Mont-Royal vers l'est au moment où un orage éclate. Supposons qu'au même moment, au FM de Radio-Canada, éclate une cantate de Bach à son paroxysme. De la buée dans le pare-brise. Tu mets ton ventilateur en route, tu baisses un peu ta vitre. La pluie rentre dans l'auto. Bon. Là, c'est Montréal. Ça va être Montréal pendant tout l'embouteillage qui va suivre, de l'avenue du Parc à la rue Rivard. C'est unique au monde. Ça devrait être classé dans le patrimone mondial de l'Unesco, en veillant à garder tous les éléments: l'orage, la cantate, la buée, l'embouteillage et le caractère québéco-indo-rocko-vidéo-sinowestern de la Mont-Royal, comment dire? Western, je pense que ça suffit. De toute façon, Montréal peut être la ville qu'on lui demande d'être. ${ }^{22}$

Prolixe, multiple, Montréal se contenterait toutefois d'une mixité non compromettante. "Que fallait-il donc pour attraper la montréalite et devenir Montréalais? " se demande un personnage d'un récit de Gaëtan Brulotte?

20 Montréal des écrivains, op. cit., p. 115.

21 Ibid., p. 38.

22 Ibid., p. 119. 
La réponse lui apparut un jour comme une évidence, à travers les multiples propos qu'on lui avait tenus et qui allaient tous dans le même sens: il était "trop" justement: trop honnête, trop appliqué, trop qualifié, trop Québécois. Eh! oui! trop Québécois pour être Montréalais. Et, surtout, il voulait trop devenir Montréalais, il était trop prêt à tout pour l'être et il avait trop de chances d'y réussir. C'est ce désir excessif qui le perdait. Ce qu'il croyait être des atouts lui nuisait à tous égards. Il fallait se montrer plus détaché, plus je-m'en-foutiste,plus n'importe quoi, plus désinvolte, pour atteindre le juste degré de sous-qualification requis. ${ }^{23}$

Ce n'importe quoi ne rappelle-t-il par le "n'importe qui" des nouvelles d'André Major?

$\mathrm{Si}$ je peux faire intervenir ici ma propre expérience, je me suis beaucoup inspirée des sollicitations qu'offre la ville dans les nouvelles de mon recueil Fugitive ${ }^{24}$. Il m'est même arrivé de trouver le lieu avant le récit. Tel café par exemple ou tel bar dans lequel j'ai observé avec minutie les déplacements des gens avant de leur inventer une histoire. La nouvelle "Times Square" est née d'un relevé méthodique et précis des messages publicitaires défilant sur Broadway. Mes histoires s'alimentent aux ondes de choc que me fournit la ville. J'affectionne tout particulièrement ce rapport du singulier au général sensible dans les lieux publics.

La nouvelle, comme tout récit, transforme le lieu en espace. Pour Michel de Certeau, après Merleau-Ponty, l'" espace est existentiel" et "l'existence est spatiale". De ce point de vue, ajoute-t-il, "il y a autant d'espaces que d'expériences spatiales distinctes". "Dans un examen des pratiques journalières qui articulent cette expérience, l'opposition entre "lieu " et "espace" renverra plutôt, dans les récits, à deux sortes de déterminations: l'une, par des objets qui seraient finalement réductibles à l'être-là d'un mort, loi d'un "lieu "; l'autre, par des opérations qui, affectées à une pierre, à un arbre ou à un être humain, spécifient des "espaces" par les actions de sujets historiques. [...] Entre ces deux déterminations, il y a des passages.... 25

23 Nouvelles de Montréal, op. cit., p. 51.

24 Lise Gauvin, Fugitives, Boréal, 1991.

25 Michel de Certeau, L'invention du quotidien, Paris, UGE, coll. "10/18", 1980, p. 209. 


\section{Art de passages, de passants, de passeurs}

La nouvelle est un art de passages, de passants, de passeurs. À ce titre, elle se rapproche de la poésie. Elle est tissage de mots, texture. Dans la nouvelle intitulée "Une femme" de Suzanne Jacob, il s'agit de dire la présence des pas qui se rapprochent en nommant trois fois le mot visage, et de donner le sujet du texte par un seul mot, le dernier, "l'accompagnant", qui oblige à une relecture de l'ensemble. La transformation qui fait basculer le récit tient au simple "passage" de l'article indéfini "une femme" à l'adjectif démonstratif "cette femme" ${ }^{26}$. Mais cette femme est aussi n'importe quelle femme, une passante, une insulaire dans la ville aux mille visages, changeante.

Proche de la modernité baudelairienne définie comme étant le "transitoire, le fugitif, le contingent", la nouvelle est aussi la forme qui, à mon avis, synthétise le mieux l'expérience formelle du modernisme et les remises en question du postmodernisme. Ne trouve-t-on pas, ici comme là, une pratique du lisible, un usage du fragment et des effets de réel dont l'impertinence apparente ne renvoie à rien de moins qu'à une interrogation sur la notion même de littérature? La nouvelle est un art urbain dans la mesure où, précisément, elle échappe à toute définition englobante, dans la mesure où elle n'est jamais totalisable. Récit d'espace, elle ne renvoie aux lieux réels que pour mieux les installer dans leur réalité de fiction. Réalité imprévisible et secrète, toujours prête à basculer dans le mystère le plus absolu. Constellation d'images, incident de frontière, la nouvelle est toujours plus ou moins, pour reprendre l'expression de Beckett, un "texte pour rien". Les auteurs de nouvelles poursuivent le rêve flaubertien d'écriture sans sujet. Ils n'en retrouvent que mieux un mode d'être existentiel, une manière d'être au monde et de le surveiller. Jaimerais ici mentionner que les titres de nouvelles sont, comme par hasard, la plupart du temps des fragments de poétique. Je viens de mentionner indirectement Le surveillant de Gaëtan Brulotte. On peut y ajouter La survie (Suzanne Jacob), La vie réelle (Gilles Marcotte), Espaces à occuper (Jean-Pierre Bergeron) et mes propres Fugitives. Alors que le conte essentialise les personnages et les lieux qu'il choisit et qui lui sont antérieurs, la nouvelle les circonscrit dans leur poids d'existence. Du côté de la phénomé- 
158

nologie, elle fait exister les choses en les disant. Si le conte est sur-écriture, la nouvelle est sur-vie. Mais elle ne décrit que pour mieux suggérer. Comme la photographie, c'" est un secret au sujet d'un secret. Plus elle vous en dit, moins vous en savez." (Diane Arbus, photographe) ${ }^{27}$.

27 Citee dans la vie en prose, juillet 1983. 